\title{
EGU21-4537
}

https://doi.org/10.5194/egusphere-egu21-4537

EGU General Assembly 2021

(c) Author(s) 2021. This work is distributed under

the Creative Commons Attribution 4.0 License.

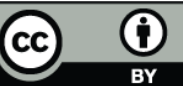

\section{Improved soil moisture estimation with Sentinel-1 for arable land at the field scale}

\author{
John Beale ${ }^{1}$, Toby Waine ${ }^{1}$, Ron Corstanje ${ }^{1}$, and Jonathan Evans ${ }^{2}$ \\ ${ }^{1}$ Cranfield University, Centre for Environmental and Agricultural Informatics, SWEE, Bedfordshire, United Kingdom of Great \\ Britain - England, Scotland, Wales (john.e.beale@cranfield.ac.uk) \\ ${ }^{2}$ UK Centre for Ecology \& Hydrology, Wallingford, Oxfordshire, United Kingdom of Great Britain - England, Scotland, Wales \\ (jge@ceh.ac.uk)
}

The change detection method of multi-temporal analysis is often used to estimate surface soil moisture from Sentinel-1 SAR data. The underlying assumptions that vegetation cover and soil surface roughness vary significantly more slowly with time than soil moisture are problematic in areas under cultivation, which are characterised by seasonal cycles of rapid crop growth, senescence, harvesting and tillage. The issue becomes more acute when data is processed at the field scale. Other areas, where the vegetation cover is persistently high, also exhibit poor sensitivity of SAR backscatter to soil moisture. In general, the mean absolute error appears to be related to the relative fractions of photosynthetically active and inactive vegetation, and bare soil. Optical indices derived from Sentinel-2 data may be used with spectral unmixing to estimate these fractions as time series at field scale. Combined with knowledge of land use, confidence levels may be assigned to each field. The soil moisture may then be estimated by two dimensional interpolation using inverse distance squared weighting across a range of neighbouring fields within a local zone. During the peak growing season, the mean absolute error in the soil moisture estimate for wheat fields is significantly reduced, in one example from around $20 \%$ volumetric water content to less than $5 \%$. This will benefit users of such products in agriculture, for example, in determining actual soil moisture deficit in the growing season. 